\title{
NEW APPROACH TO FULFILL ART 8 OF DIRECTIVE 2009/128: A RISK ASSESSMENT PROCEDURE FOR PESTICIDE APPLICATION EQUIPMENT
}

\author{
Marie STAS $^{1}$, David NUYTTENS ${ }^{2}$, Olivier MOSTADE ${ }^{1}$, Johan DECLERC ${ }^{2}$, \\ Ingrid ZWERTVAEGHER ${ }^{2}$, Guillaume DEFAYS ${ }^{1}$, Donald DEKEYSER ${ }^{2}$, \\ Bruno HUYGHEBAERT ${ }^{1}$ \\ ${ }^{1}$ Walloon Agricultural Research Center (CRA-W), Agricultural Machines and Facilities Unit, Gembloux, \\ BELGIUM \\ ${ }^{2}$ The Institute for Agricultural and Fisheries Research (ILVO), Technology and Food Science Unit - \\ Agricultural Engineering, Merelbeke, BELGIUM \\ E-mail of corresponding author: m.stas@cra.wallonie.be
}

Keywords: pesticide application equipment, inspection, risk assessment, guidelines, exemption.

\begin{abstract}
All pesticide application equipment in professional use shall be subject to inspections at regular intervals according to the EU Directive 2009/128/EC on the sustainable use of pesticides. Article 8.3 allows the Member States to derogate from the mandatory inspection at regular intervals or to apply different timetables and inspection intervals for certain types of pesticide application equipment based on a risk assessment for human health and environment and an assessment of the scale of use. In order to fulfill Article 8.3, a risk assessment protocol was developed in Belgium within the framework of the SIRAAPESTICON project. It is then applied on the Belgian equipment in use. Therefore, risk is evaluated for the human health and the environment. The assessment is based on technical parameters subject to inspections, their occurrences and severities, but also on national scale of use of the PAE types. Results are expressed at different scale levels: the defect, the machine and the country. They also can be used to help in elaboration of new inspection protocols. This new procedure offers guidelines about the necessity to carry out an inspection of every PAE in use.
\end{abstract}

\section{INTRODUCTION}

The EU Directive 2009/128/EC on the sustainable use of pesticides requires that Member States (MS) shall ensure that all Pesticide Application Equipment (PAE) in professional use shall be subject to inspection at regular intervals (Art. 8.1 and 8.2). However, Article 8.3 of the Directive allows the MS to derogate from the mandatory inspection at regular intervals or to apply different timetables and inspection intervals for certain types of PAE based on a Risk Assessment (RA) for human health and environment and an assessment of the Scale of Use. The RA process should demonstrate the usefulness of the inspection to significantly decrease the risk of the use of the PAE. The Belgian method from the SIRA-APESTICON project defines the risk by a combination of two factors: 1 . the severities of harm on exposed subjects and 2. the occurrences of hazard. In the context of this work, harm is the consequence of technical defects: over-dosage, under-dosage, or injuries induced by the use of PAE during the pesticide applications. Occurrences of defects are defined by PAE technical inspection. Risk is calculated for the health of the operator, the health of the consumer and for the environment. This paper shows an overview of the results for Belgium.

\section{MATERIALS AND METHODS}

Risk is the result of the combination of occurrence and severity of harm: In this case, occurrence is relative to PAE technical defect. They were extracted from the data of 2011, 2012 and 2013 of the Belgian inspection services. Harms result of the hazard and of the way of exposure. In this case, this can be over- or under-dosages or injuries induced by the use of PAE presenting technical defects. Values of severities of harm were defined 
based on an international enquiry submitted in particular to European experts from the SPISE (Standardized Procedure for the Inspection of Sprayers in Europe) community.

The risk calculation was made using two methods to combine the severity of harm and the occurrence: "Defects only" or "Defects + residual risk". Formulations are given in Table 1. The "residual risk" is the risk induced by potential undetectable (at the inspection) small deficiencies combined with the risk inherent to the use of PAE even without any defect. Risks are calculated at three different scales: 1 . the defect individually, 2. the entire machine (sum of defect's risks) and 3. the country or for all machines of a given PAE type on the national territory, thanks to the factor of scale of use. These scales of use were based on a combination of the frequencies of use (obtained by a national enquiry) of the different PAE types and the weight $(\mathrm{kg})$ of pesticide potentially applied. Sales of active substances in Belgium $(\mathrm{kg})$ were selected for the years 2011, 2012 and 2013 from the Eurostat database. An estimation of the human part of risk (behaviour of the operator) can be added to the technical risk to obtain a total risk of pesticide application. Therefore, the partition between the technical part and the human behaviour part of risk (\% of a total risk) was determined by an enquiry submitted to European experts. Each risk calculation is performed "before" and "after inspection". In one hand, "Before inspection" illustrates the presence of a defect, above tolerance level of inspection and without defect correction. In another hand, "After inspection" illustrates a defect repaired regarding the inspection tolerance level or an absence of defect. The objective is to evaluate the potential risk reduction induced by the inspection.

Table 1. Calculations used to apply the RA in Belgium. Two methods: Defects only and Defects+residual risk. Scales: defect, machine, Belgium, Belgium+human part. Two situations: before technical inspection and after technical inspection

\begin{tabular}{|c|c|c|}
\hline Method & $\begin{array}{c}\text { Calculation before technical } \\
\text { inspection }\end{array}$ & $\begin{array}{c}\text { Calculation after technical } \\
\text { inspection }\end{array}$ \\
\hline $\begin{array}{l}\text { "Defects only" at the } \\
\text { scale of the defect }\end{array}$ & $\begin{array}{l}\text { occurrence }^{*} \text { severity } \\
=\text { Risk }_{\text {defect }} \text { before }\end{array}$ & $\begin{array}{l}\text { occurrence }^{*} \text { severity } \\
=\text { Rister }_{\text {defect.after }}\end{array}$ \\
\hline $\begin{array}{l}\text { "Defects only" at the } \\
\text { scale of the machine }\end{array}$ & $\begin{array}{l}\sum \text { Risk }_{\text {defect.before }} \\
=\text { Risk }_{\text {machine.before }}\end{array}$ & $\begin{array}{l}\sum \text { Risk }_{\text {defect.after }} \\
=\text { Risk }_{\text {machine.after }}\end{array}$ \\
\hline $\begin{array}{l}\text { "Defects + residual } \\
\text { risk" at the scale of } \\
\text { Belgium }\end{array}$ & $\begin{array}{l}\left(\sum R_{\left.i s k_{\text {defect }+ \text { resi.before }}\right)}{ }^{*} \text { scale_of_use }\right. \\
=\text { Risk }_{\text {Belgium.before }}\end{array}$ & $\begin{array}{l}\left(\sum \text { Risk } k_{\text {defect }+ \text { resi.after }}\right)^{*} \text { scale_of_use } \\
=\text { Risk }_{\text {Belgium.after }}\end{array}$ \\
\hline $\begin{array}{l}\text { "Defects + residual } \\
\text { risk" at the scale of } \\
\text { Belgium, + human part }\end{array}$ & $\begin{array}{l}\left(\text { Risk } k_{\text {defect+resi.before }}\right) * \text { scale_of_use*par } \\
\text { tition_human_vs_technical_part } \\
=\text { Risk }\end{array}$ & $\begin{array}{l}\left(\text { Risk } k_{\text {defect+resi.after }}\right) * \text { scale_of_use*part } \\
\text { ition_human_vs_technical_part } \\
\text { = Risk }\end{array}$ \\
\hline
\end{tabular}

\section{RESULTS AND DISCUSSION}

\section{Defects only, scale of the defect}

At the scale of the defect, risk values are very useful to elaborate new inspection protocols. As they are defined before and after inspection, they inform about the risk reduction obtained by the inspection of every individual parameter. Differences in risk values are observed between defects meaning that some defects give harms more severe than others and/or that some defects occur more often than other. For a given defect, the risk varies from a PAE type to another with the variation of occurrences between PAE types. The defects for which the risk before inspection is close to zero could be exempted from inspection. By example, for the pressure deviation because of a defect in a section, the risk is below 0.025 before inspection. The defects that present the biggest risks should be inspected. For example, the readability of the tank content indicator has risks values 
around 1 before inspection. The decision about inspection of a defect can also be influenced by the risk reduction induced by inspection. For example, the risk reductions induced by the replacement of an absent tank filling strainer are among the most important ( $-67 \%$ for the operator). In another hand, for example the nozzles individual flow rate has lowest risk reductions ( $-37 \%$ for the operator).

\section{Defects only, scale of the machine}

A direct effect of inspection can be observed with the method "defects only". Analysis at the scale of the machine could be useful to evaluate the risk for the operator who is exposed to only one machine at a time. A graphic illustration is given for knapsack sprayers in Figure 1. Variations of risk values can be noticed between subjects at risk. Relative risk reduction are calculated on the risk "after inspection" as a percentage of the risk value "before inspection". The type of defects listed can influence the final risk value because the severities of harm can be higher for some defects than for other defects and because the occurrence can also be higher. The risk reductions at the scale of the machine have to be subject of attention because they directly reflect the effects of a technical inspection. In the case of Belgium, a clear effect of inspection is observed on risk results (risk reduction of about 63\%; Figure 1). For decision making, a maximum level of risk could be defined for the operator safety and another maximum level of risk can be defined for exclusion of inspection. Before to be fixed, the maximum values should be subject of discussion by decision makers with taking account of the other risk results (at the scale of the country), of the scale of use and, when justified, of the toxicity of substance applied.

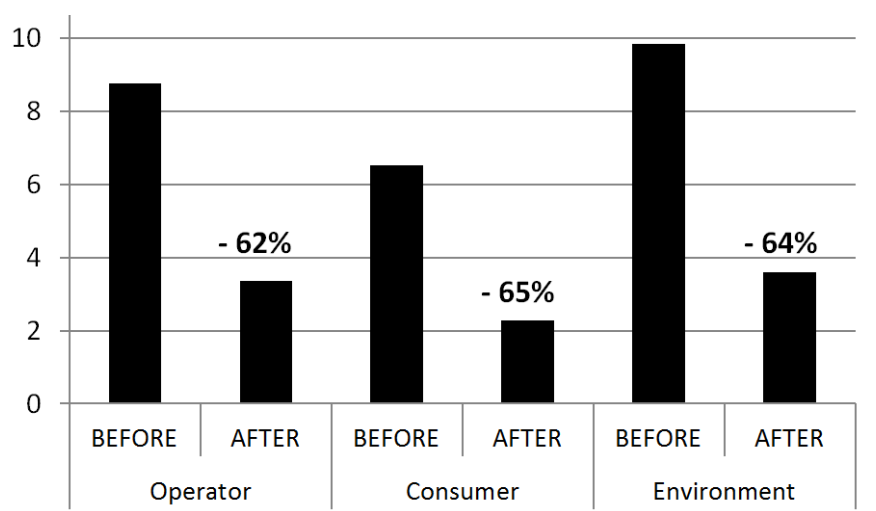

Figure 1. Results of risk calculation in absolute values for Knapsack sprayers. Risks for the operator, for the consumer and for the environment. Scale of the machine, method "defects only". BEFORE=before inspection; AFTER=after inspection. Risk reduction between BEFORE and AFTER are indicated in percentage.

\section{Defects + residual risk, scale of Belgium (technical risk)}

The advantage of the analysis of technical risk at Belgian scale is to obtain a global view on the total technical risk for one PAE type or to compare different PAE types (Figure 2). The differences of risk values between PAE types are mainly due to the scales of use that are specific to each PAE type. In Belgium the field crop sprayers have the biggest scale of use with $78 \%$ of the total scale of use. The orchard, knapsack and fixed and semi mobile sprayers have scales of use corresponding respectively to $5 \%, 7 \%$ and $4 \%$ of the total. Percentages of risk reductions with the method ("Defect only+residual risk") are smaller $(\sim 10 \%)$ than with the method "Defects only" ( $\sim 65 \%)$. This observation is explained by the fact that "residual risks" is equally distributed between "before" and "after inspection" and take a big part in the total risk. Absolute values of risk are above 10000 (results of 
calculation haven't standard unit). The national scale is interesting concerning the risk for the consumer and the risk for the environment because they are targeted by pollution related to broad crop surfaces. For decision making, a maximum level of risk can be tolerated excluding inspection of certain types of PAE.

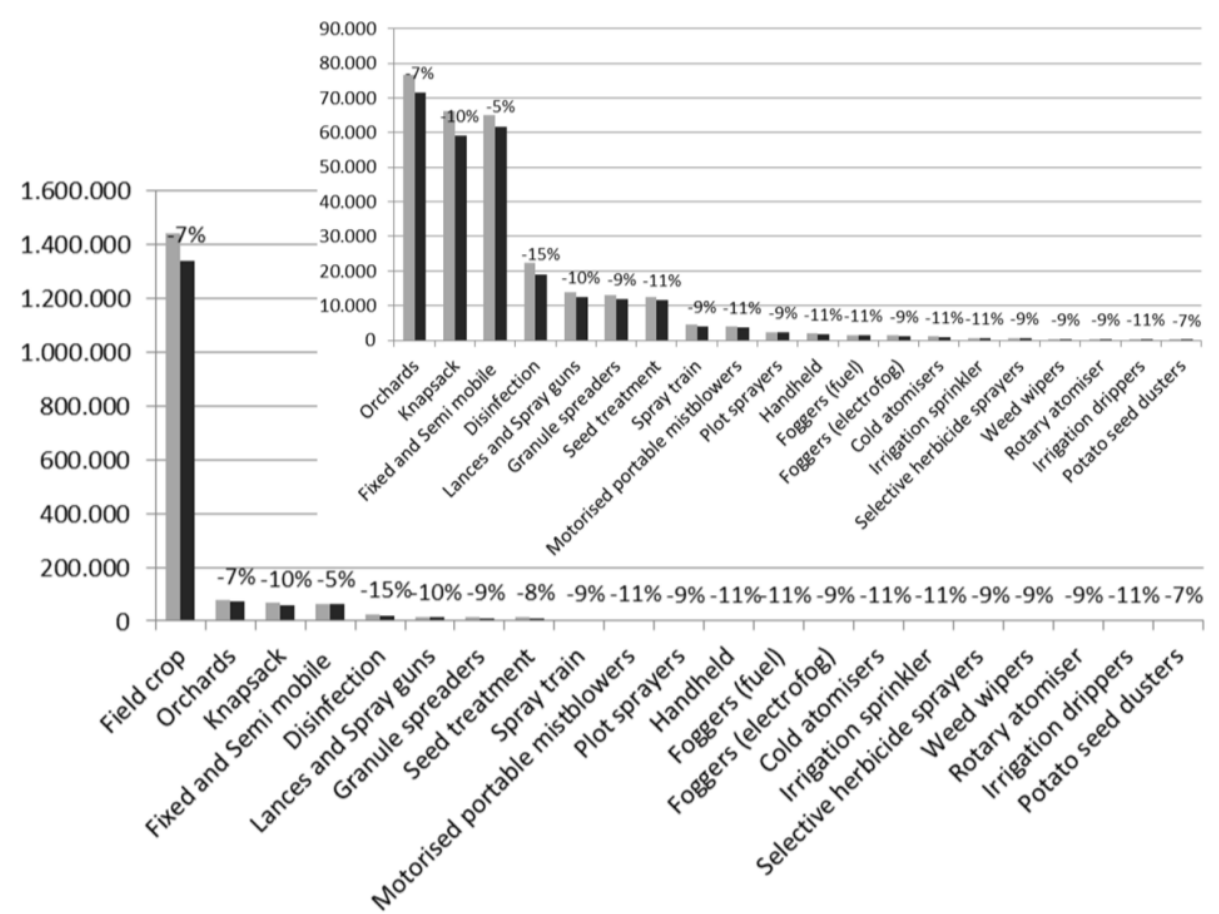

Figure 2. Risks calculated by the method "Defects only+residual risk" at the scale of Belgium for different PAE types (technical risk). Risk values are average of risk for the operator, risk of the consumer and risk for the environment. Grey: risk values before technical inspection; Black: risk values after technical inspection; Values in percentage: risk reductions between the risk before and risk after technical inspection

\section{Defects + residual risk, scale of Belgium (technical and human part of risk)}

This last method of risk calculation is interesting to compare pesticide application of the different PAE types (Figure 3). That is the most complete, including defects and residual technical risks, scale of use and human behaviour part of risk. As previously, differences in risk values between PAE types are mainly due to the scale of use. However, risks related to pesticide application are, for some PAE types, more dependent of user's behaviour (e.g.: knapsack sprayers). For other PAE types, as spray train or irrigating systems, the technical part of risk is more dominating. The percentage of risk reduction is very low $(\sim 5 \%)$ because the residual risk and the human part of risk are added equally to the risks value before and after inspection. The partition between human and technical part of risk could justify the inspection in order to significantly reduce the total risk. 


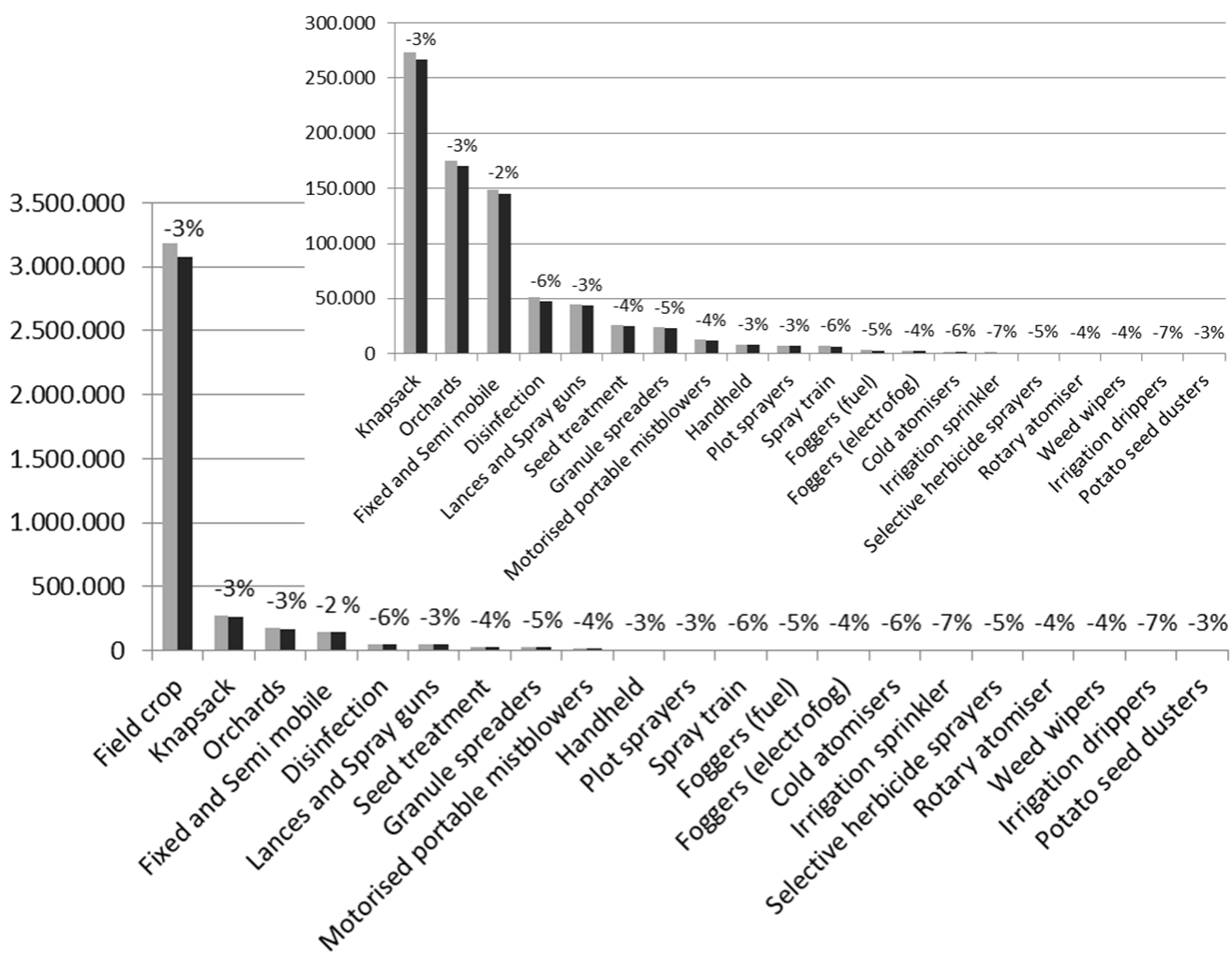

Figure 3. Risks calculated by the method "Defects only+residual risk" at the scale of Belgium for different PAE types and added to the human part of risk (technical risk + human risk). Risk values are average of risk for the operator, risk of the consumer and risk for the environment. Grey: risk values before technical inspection; Black: risk values after technical inspection; Values in percentage: risk reductions between the risk before and risk after technical inspection

\section{CONCLUSION}

Results of the method applied in Belgium illustrate the effects of inspection of each PAE type and the variation of scales of uses between all of them. Risks can be calculated for an unlimited number of PAE types. Risk reductions give indication on potential efficiencies of inspection for all PAE types. It distinguished different targets (operator, consumer, and environment) and the results can be obtained at different scales of calculation (the technical defect, the machine or the scale of the country).Absolute values of the risk increase with the scales of calculation. They are around 0-1.5 for the scale of the defect, until 13 for the scale of the machine, until 1500000 for the scale of Belgium and until 3250000 for the scale of Belgium with the addition of the human part of risk. Risk reductions expressed in percentage are similar trough all PAE types but absolute values of reduction are proportional to absolute value of risks that vary between PAE types. These last are mainly due to the complexity of the PAE and its inspection protocol when analysis is made at the scale of the machine. On the other hand, they are mainly due to scale of use when analysis is made at the scale of Belgium. Risk values and risk reduction values at every step of the risk assessment are strong theoretical basis to support decision making. Regarding risk results, PAE types already inspected in Belgium are those for that inspections are the most useful (field crop, orchard, fixed and semi mobile, disinfection equipment). 


\section{REFERENCES}

Directive (CE) $n^{\circ} 2009 / 128$ of the european parliament of the council of 21 october 2009 establishing a framework for Community action to avieve the sustainable use of pesticides, O.J.E.U., L.309/71, November, 242009.

European Commission. Eurostat - Your key to European statistics. [Online] [Cited: March 21, 2017.] http://ec.europa.eu/eurostat/data/database.

EFSA. (2013). International Frameworks Dealing with Human Risk Assessment of Combined Exposure to Multiple Chemicals. Parma, Italy: EFSA Journal.

FAO. (2015). Progress in pesticide risk assessment and phasing-out of highly hazardous pesticides in Asia. Bangkok, Thailand: Piao Yongfan.

Ganzelmeier, H. (2012). Risk assessment for human health and the environment. Fourth European Workshop on Standardised Procedure for the inspection of sprayer - Lana (South Tyrol): SPISE Working Group. SPISE 4, 94-100.

Gil, Y. S. (2005). Emission of pesticides to the air during sprayer application: A bibliographic review. Atmospheric Environment, 39, 5183-5193.

Health Council of the Nederlands. (2014). Crop protection and local residents. Health council of the Nederlands.

IPCS. (2009). Principles and methods for the risk assessment of chemicals in food. World Health Organization, FAO. Ottawa, Canada: Marla Sheffer.

IPCS. (2010). WHO Human Health Risk Assessment Toolkit: Chemical Hazards. Ottawa, Canada: World Health Organization.

ISO 12100 (2010) Safety of machinery - General principles for design - Risk assessment and risk reduction, C ISO $2010-78 \mathrm{p}$.

Marot, J. R. (2008). Contribution à l'actualisation des indicateurs de l'état de l'environnement wallon relatifs à l'utilisation des produits pharmaceutiques. UCL, Unité de phytopathologie, Louvain-la-Neuve, Belgique.

Nohl, J. T. (1988). Systematik zur Durchführung von Gefährdungsanalysen. Bremerhaven: Wirtschaftsverlag NW.

Phytophar. (2012). Rapport d'activités . Oostkamp, Belgium.

Roettele, M. B. (2011). EOS handbook. Environmentally Optimized Sprayer. Background and Documentation. TOPPS.

Stas, M., Nuyttens, D., Zwertvaegher, I., Defays, G., Declercq, J., Mostade, O., Dekeyser, D., Huyghebaert, B., 2016. Development of a risk assessment procedure for pesticide application equipment within the framework of article 8 of the EU Directive 2009/128 and development of specific inspection procedures. APESTICON final report, 'FOD Volksgezondheid, veiligheid van de voedselketen en leefmilieu' (RT 14/8 Apesticon 1), December 2016.

Stas, M., Nuyttens, D., Zwertvaegher, I., Defays, G., Declercq, J., Mostade, O., Dekeyser, D., Huyghebaert, B., 2016. Development of a risk assessment procedure for pesticide application equipment within the framework of article 8 of the EU Directive 2009/128 and development of specific inspection procedures. Communications in Agricultural and Applied Biological Sciences. Ghent University, Belgium.

Vercruysse, F. S. (2002). POCER, the pesticide occupational and environmental risk indicator. Crop Protection, 21, 307-3015.

Vergucht, S. C. (2006). "Belgian Pesticide Risk and Use Indicators Methodology" Synthèse. Gand, Belgium: UGent.

Wegener, J. (2013). Application of the Zurich methodology for risk assessment according to article 8(3) of sustainable use directive (inspection of equipment in use) at example of German. 5th european workshop on standardised procedure for the inspection of sprayers in Europe-SPISE 5. Braunschweig, Germany.

WHO. (2009). Principles and methods for the risk assessment of chemicals in food. Chapter 2: Risk assessment and its role in risk analysis. (IPCS, Éd.) Environmental Health Criteria, 240. 